\title{
The impact of intraoperative blood pressure variability on the risk of postoperative adverse outcomes in non-cardiac surgery: a systematic review
}

\author{
Zbigniew Putowski ${ }^{1}$ Marcelina Czok ${ }^{1} \cdot$ Łukasz J. Krzych $^{2}$
}

Received: 30 September 2021 / Accepted: 31 December 2021 / Published online: 13 January 2022

(c) The Author(s) 2022

\begin{abstract}
Hemodynamic stability during surgery seems to account for positive postoperative outcomes in patients. However, little is known about the impact of intraoperative blood pressure variability (IBPV) on the postoperative complications. The aim was to investigate whether IBPV is associated with the development of postoperative complications and what is the nature of this association. We conducted a systematic search in PubMed, Medical Subject Headings, Embase, Web of Science, SCOPUS, clinicaltrials.gov, and Cochrane Library on the 8th of April, 2021. We included studies that only focused on adults who underwent primarily elective, non-cardiac surgery in which intraoperative blood pressure variation was measured and analyzed in regard to postoperative, non-surgical complications. We identified 11 papers. The studies varied in terms of applied definitions of blood pressure variation, of which standard deviation and average real variability were the most commonly applied definitions. Among the studies, the most consistent analyzed outcome was a 30-day mortality. The studies presented highly heterogeneous results, even after taking into account only the studies of best quality. Both higher and lower IBPV were reported to be associated for postoperative complications. Based on a limited number of studies, IBPV does not seem to be a reliable indicator in predicting postoperative complications. Existing premises suggest that either higher or lower IBPV could contribute to postoperative complications. Taking into account the heterogeneity and quality of the studies, the conclusions may not be definitive.
\end{abstract}

Keywords Variability $\cdot$ Blood pressure $\cdot$ Intraoperative period $\cdot$ Postoperative complications

\section{Introduction}

Intraoperative hemodynamic homeostasis remains one of the crucial factors accounting for the postoperative prognosis of patients undergoing either cardiac or non-cardiac surgery [1]. Blood pressure monitoring stands as a cornerstone method for assessing perfusion during surgery [2]. The relationship between intraoperative hypotension $(\mathrm{IOH})$ and postoperative complications received much attention during

Zbigniew Putowski

putowski.zbigniew@gmail.com

1 Students' Scientific Society, Department of Anaesthesiology and Intensive Care, Faculty of Medical Sciences in Katowice, Medical University of Silesia, Medyków 14 Street, 40752 Katowice, Poland

2 Department of Anaesthesiology and Intensive Care, Faculty of Medical Sciences in Katowice, Medical University of Silesia, Katowice, Poland the recent years; however, there are still various aspects of intraoperative blood pressure that could enhance our understanding of tissue perfusion [3]. One of these aspects is the intraoperative blood pressure variability (IBPV). IBPV is a continuous variable that describes the degree of variation of a set of blood pressure measurements. It can be expressed in various forms, an example of which is the standard deviation (SD). On one hand, too high IBPV could reflect hemodynamic instability, i.e., successive changes in blood pressure would result in simultaneous perfusion disturbances (even without directly experiencing hypo- or hypertension). On the other hand, too low IBPV could reflect a patient's inability to adapt to changing hemodynamic circumstances (e.g., persistent hypotension despite adequate fluid infusion or vasopressor support would result in low IBPV). Moreover, in 2019, the Perioperative Quality Initiative (POQI) consensus on intraoperative blood pressure cited only one paper that focused on IBPV [1]. Due to seemingly limited data on the above-mentioned issue and unclear physiological 
implications, we decided to collect and analyze all the available data regarding this interesting issue in a systematic manner. Thus, this review aimed to answer the following question: If IBPV impacts postoperative outcomes, what is the nature of this association?

\section{Methods}

This study was conducted in accordance with the PRISMA 2020 checklist [4].

\section{Eligibility criteria}

We included studies that only focused on adults who underwent primarily elective, non-cardiac surgery in which IBPV was measured and analyzed in regard to postoperative, nonsurgical complications. We included papers of which full reports were published before the day of search. Additionally, the papers had to be published in English, regardless of the year of publication. Studies were excluded when they selected a subgroup of patients with a specific comorbidity that was not part of the reason to perform the surgical procedure. Additionally, cardiac surgery papers, papers that included only emergency procedures, case reports, case series, systematic reviews, and meta-analyses were excluded. Studies in which blood pressure measurements were performed in time intervals longer than 5 min were not taken into account as well, as in such cases IBPV could have been omitted in a more significant matter.

\section{Information sources}

The search was conducted within PubMed, Medical Subject Headings, Web of Science, SCOPUS, clinicaltrials.gov, Embase, and Cochrane Library on the 8th of April, 2021.

\section{Search}

Search string for PubMed: (((intraoperative OR intraoperatively OR perioperative OR perioperatively) AND (variation OR lability OR variability OR deviation OR coefficient of variation OR fluctuation)) AND (myocardial injury OR major adverse cerebrovascular cardiovascular events OR postoperative complication* OR adverse outcome* OR cardiac OR renal OR organ injury OR organ dysfunction OR acute kidney injury OR myocardial infarction OR stroke OR death OR mortality OR length of stay OR cerebral OR complication OR adverse event* OR ischaem* OR injury OR delirium OR cerebrovascular OR coronary OR LOS OR accident)) AND (blood pressure OR systolic blood pressure OR diastolic blood pressure OR mean arterial pressure).
The remaining search strings are available in the Supplementary material 1 .

\section{Study selection and data collection process}

After importing all the papers from the initial search using search string, two independent investigators assessed studies by analyzing titles and abstracts (via Mendeley ${ }^{\circledR}$ ). This study was processed further if all adjudicators (ZP and MC) agreed to include the paper for review. If only one reviewer agreed to proceed with the manuscript, the second assessment of the paper was performed by the third investigator (ŁJK).

\section{Data items}

Authors, year of publication, type of a study, patients' characteristics, type of surgery, intraoperative blood pressure variability, and postoperative complications were outcomes.

\section{Quality assessment}

Newcastle-Ottawa scale (NOS) was implemented to assess the quality of the included studies [5]. The total NOS score of each study was converted to Agency for Healthcare Research and Quality standards [6]. In regard to the "comparability" criteria, we considered that the most important variable that needed to be controlled for was the occurrence of IOH. Two independent investigators (ZP and MC) performed the quality assessment and then, any differences were resolved by a discussion and the final decision was accepted by ŁJK.

\section{Results}

\section{Included studies}

By using the search string within various medical databases (look at Information sources), we identified 2949 articles in total. After removing duplicates $(n=470)$, we screened the remaining papers by evaluating titles and abstracts $(n=2479)$. By using the PICO criteria and the inclusion and the exclusion criteria, we distinguished 97 papers for the full-text read assessment. After excluding the articles for numerous reasons (non-English papers $=2$, papers not complying with the PICO criteria $=79$, and papers with no full-text available $=5$ ), the final 11 papers were included in the systematic review. There were 9 cohort studies $(6$ retrospective $[7,8,12,14,15,17] 3$ prospective studies [11, $13,16])$ and 2 case-control studies $[9,10]$. Study selection process is presented on a flowchart (Fig. 1). 


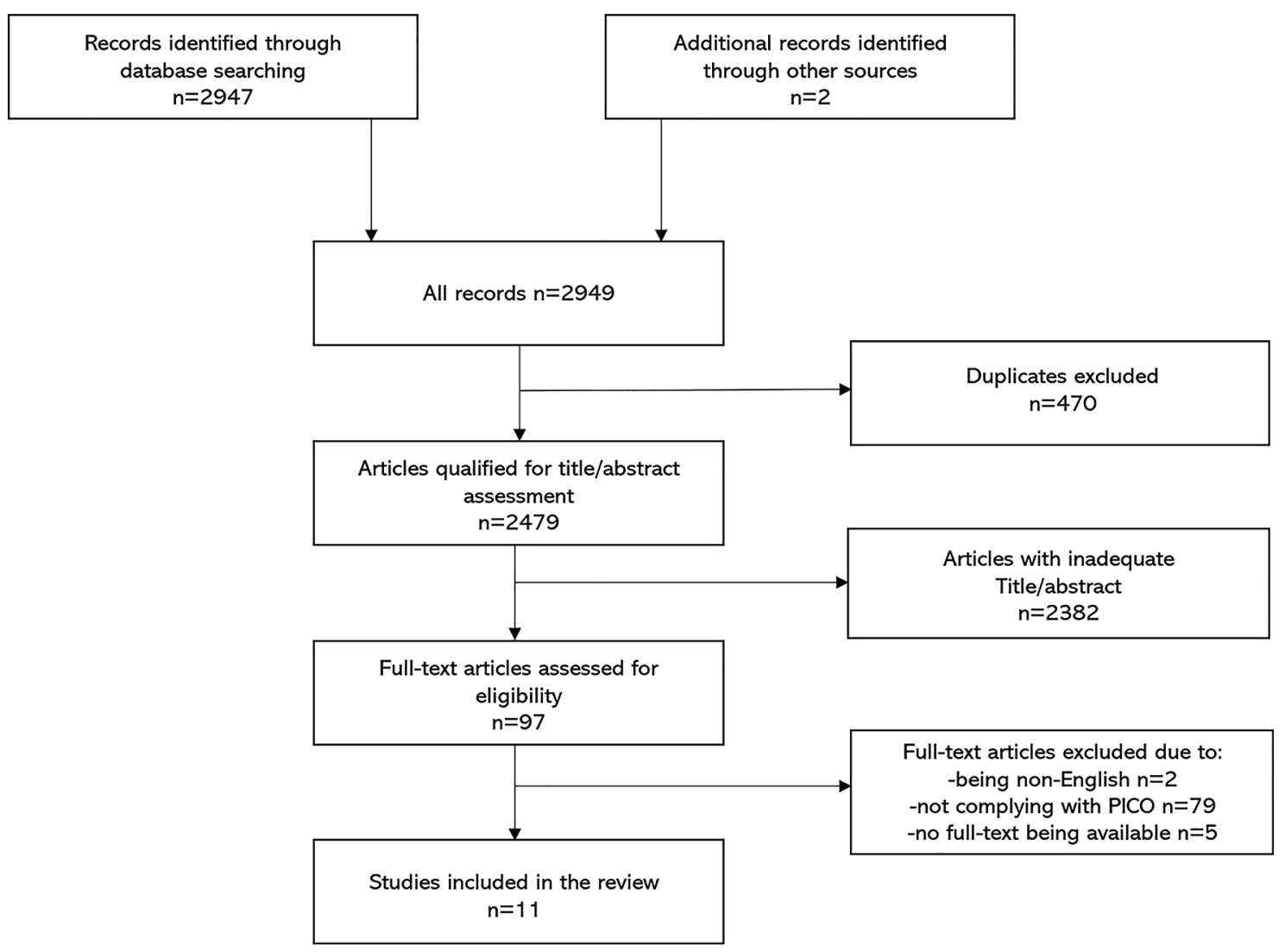

Fig. 1 Flowchart of the progress of retrieved reports through the review

\section{Quality assessment}

By implementing NOS, we assessed all 11 studies (of which 9 were cohort studies and 2 were case-control studies). Overall, we identified 4 studies of "good" quality [11, 12, $15,17]$ and 7 of "poor" quality (Supplementary material 2) [7-10, 13, 14, 16]. The quality of the latter studies suffered from the lack of adequate controlling for confounding factors, as these studies did not control for IOH. In general, there were no systematic problems regarding "selection" and "outcomes" criteria.

\section{Patient characteristics}

The number of patients varied from 33 to 104,401, with a median of 917 patients (Table 1 and Supplementary material 3) [7-17]. The mean or the median age of participants in 7 studies was below 65 years [10, 12-17], whereas in the remaining 4 , above 65 years [7-9, 11]. Information regarding gender was available in 9 studies [7-10, 12-17], of which 6 had a similar gender ratio of about 40-60\% [7, $8,10,12,15,17]$. In 4 studies, all patients underwent general anesthesia $[7,10,14,16]$, in the remaining 5 studies patients received either regional or general anesthesia [9,
11-13, 17], whereas in 2 studies no information regarding anesthetic method was provided (Supplementary material 3) $[8,15]$. All patients underwent non-cardiac surgery [7-17]; however, in 2 studies, no "type of surgery" information was provided [7, 8]. 3 studies analyzed neurointerventional patients $[9,10,16], 1$ study analyzed liver transplantation patients [14], 1 study analyzed orthopedic surgery patients [11], whereas in the remaining 4 studies, patients underwent various non-cardiac procedures, of which abdominal and orthopedic surgeries were the most common $[12,13,15,17]$. In regard to frequency of procedural electiveness, 5 studies included patients who underwent only elective surgery [7, 9, 10, 14, 16]; 5 studies had mixed populations of patients undergoing elective and non-elective procedures [8, 12, 13, 15, 17]. In one study, the data regarding emergent procedures were not provided (Supplementary material 3) [11].

IBPV, intraoperative blood pressure variation; IQR, interquartile range; MAP, mean arterial pressure; SBP, systolic blood pressure; DBP, diastolic blood pressure; MAD, median absolute deviation; POD, postoperative delirium; OR, odds ratio, 95\% CI, 95\% confidence interval; SD, standard deviation; CV, coefficient of variation; ARV, average real variability 


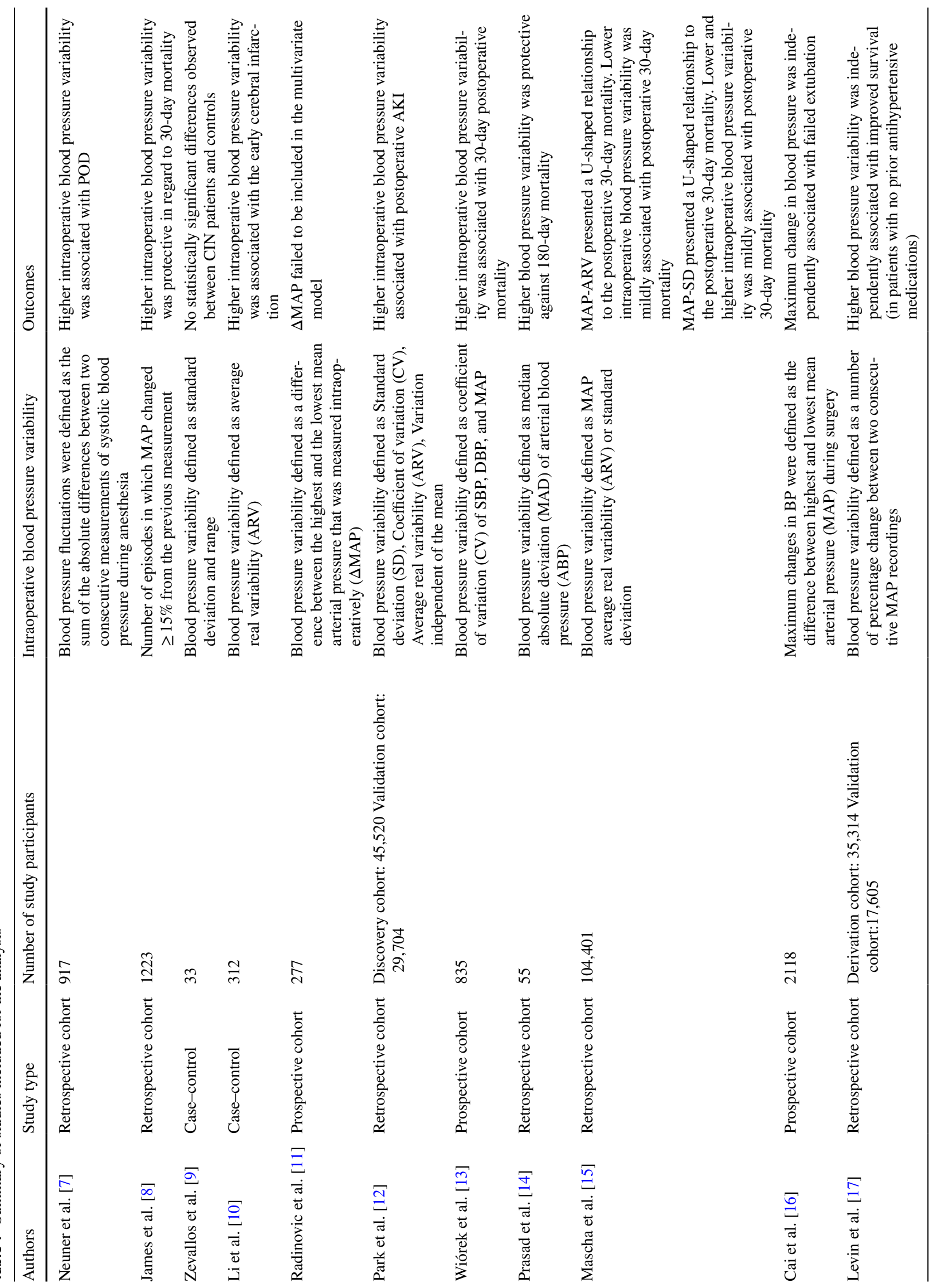




\section{Blood pressure variability definitions}

Blood pressure component that was studied the most was the mean arterial pressure $(n=10)$ [8-17], then systolic blood pressure $(n=4)$ [7, 9, 10, 13] and diastolic blood pressure $(n=3)$ (Table 1) $[9,10,13]$. Blood pressure variability definitions varied between the studies: 5 studies implemented more than one definition of blood pressure variability [9, $10,12,13,15]$, whereas the other 6 studies introduced only one definition [7, 8, 11, 14, 16, 17]. The most consistent definitions included a standard deviation $(n=4)[9,12,14$, $15]$, a coefficient of variation $(n=2)[12,13]$, an average real variability $(n=3)[10,12,15]$, and a variability defined as a difference between the highest and the lowest measured MAP $(n=2)[11,16]$. The remaining studies implemented different definitions [7, 8, 17] (Table 1).

\section{Outcomes}

Mortality was the most frequently investigated outcome among the included studies $(n=6)[8,12-15,17]$, of which 30 -day mortality was the most frequent $(n=4)[8,13,15,17]$ (Table 1 and Supplementary material 3). The occurrence of 7 -day postoperative delirium was described in 2 papers [7, 11]. The remaining studies focused on different aspects of postoperative complications, including acute kidney injury [12], contrast induced nephropathy [9], early cerebral infarction [10], and failed extubation [16].

\section{A relationship between IBPV and outcomes}

In 5 studies, a relationship between higher intraoperative blood pressure variability and postoperative complications was observed [7, 10, 12, 13, 16]. In 3 studies, the authors discovered a protective effect of higher blood pressure variability on the risk of postoperative complications [8, 14, 17$]$. One study observed a U-shaped relationship between blood pressure variability and postoperative complications [15]. In the 2 remaining studies, no association between the variable and the outcome was observed $[9,10]$.

\section{Discussion}

This systematic review focused on summarizing the data regarding intraoperative blood pressure variability and postoperative complications (as of April 2021). To our knowledge, this is the first attempt to systemize the body of evidence of the issue. No clear message regarding the nature of the relationship between IBPV and postoperative outcomes can be produced.

There were many different IBPV definitions applied across the studies. A standard deviation (SD) was the most frequently implemented definition of IBPV. SD reflects a spreadness of data from the mean value of all recordings; however, it does not take the mean value into account. An alternative then is to use a coefficient of variation (CV) which normalizes SD to the mean. For example, the same value of SD (e.g., 5) for two different means (10 and 100) results in dramatically different CVs: $50 \%$ and 5\%. However, both $\mathrm{CV}$ and SD provide a global value of variation, irrespectively of measure-to-measure recordings. Hansel et al, provided an equation for an average real variability (ARV) which takes into account the latter [18]. They have shown that ARV can estimate blood pressure variability better than SD. Mascha et al., however, recognized that ARV is only valid in regard to equally distant time of measurements [15]. Therefore, they provided a generalized ARV formula which did not require equal time intervals between the recordings. ARV (and generalized ARV) was implemented in 3 studies. Due to above-mentioned reasons, we suggest using ARV as a standard measure of blood pressure variability. However, we are unable to make a clear recommendation regarding the physiological range of ARV as the authors present contrary results and, additionally, none of them provided a specific cut-off point for this parameter.

The studies of the best quality presented highly heterogeneous results $[11,12,15,17]$. Therefore, no clear message regarding the relationship between IBPV between postoperative outcomes can be produced. The biggest methodological obstacle to overcome in regard to high variability is to understand the confounding effects of either hypo- or hypertension. Theoretically, the same IBPV values can result from two very different ranges of blood pressure, e.g., IBPV that occurs below hypotensive thresholds of blood pressure versus IBPV that occurs within physiological range of blood pressure. Indeed, in Park's study of 29,704 patients (a validation cohort), a high IBPV was associated with postoperative AKI in a meaningful way only when the high variability occurred below $65 \mathrm{mmHg}$ of MAP [12]. This finding contributes to the hypothesis that deeper variations (e.g., below certain thresholds) of BP may indeed be more harmful than little, more frequent IBPVs that would result in the same value of variability. Moreover, this could explain why in Wiórek's, Cai's, and Li's studies, a higher degree of variability of MAP was associated with 30-day mortality: the authors did not adjust for intraoperative hypotension; therefore, a higher IBPV could reflect the occurrence of hypotension $[10,13,16]$. Nevertheless, a high IBPV, irrespective of the occurrence of hypotension, could result in perfusion disturbances, as rapid changes in blood pressure may exceed the capacity of adaptation and thus may not be simultaneously followed by sufficient neurohormonal and vascular response [12, 19]. Therefore, theoretically, frequent changes of blood pressure within the physiological range could still lead to imbalance of perfusion, albeit not 
exceeding hypotension thresholds. Two studies of the largest populations (Mascha $=104,401$ patients, Levin $=52,919$ patients), despite implementing different IBPV definitions, (ARV for Mascha and MAP lability $>10 \%$ for Levin) concluded that higher variability was associated with 30-day postoperative survival $[13,15]$. Interestingly, in Mascha's study, they observed a U-shaped relationship between ARV and mortality. By taking into account a number of potentially confounding factors (including time-weighted average MAP), they provided conclusions that low IBPV was associated with 30-day mortality and that low IBPV could reflect the impaired circulatory system's ability to adapt to the hemodynamic disturbances, and thus, patients with low IBPV would be more susceptible to such alterations and be unable to restore homeostasis, and, therefore, have a higher risk of insufficient perfusion. Such a hypothesis was based on considerations that low heart rate variability is a marker of autonomic dysfunction in patients with heart failure and could predict cardiovascular events [15, 20]. Moreover, anesthesia depth may play a role in shaping the IBPV as excessive doses of anesthetic agents interfere with the autonomic system response, often leading to sympathetic depression [21]. It is likely that depth of anesthesia influences hemodynamic stability; however, as of now, there are no studies assessing this issue in regard to postoperative complications.

In terms of limitations, the data covered by this review are very heterogeneous in regard to study populations, IBPV definitions, and outcomes. Therefore, we failed to prepare a reliable meta-analysis. Secondly, the studies varied in terms of quality of reporting. Thirdly, all the studies included in the review were observational studies; therefore, we cannot imply causality of IBPV on postoperative outcomes. Taking into account all of the above-mentioned considerations, we believe that unification of IBPV definition is the first step to ultimately determine the role of IBPV. Further studies exploring this parameter, especially within the physiological BP thresholds, could expand our knowledge regarding its influence on perfusion disturbances.

\section{Conclusion}

To conclude, based on a limited number of studies, IBPV does not seem to be a reliable indicator in predicting postoperative complications. The primary question of this systematic review cannot be clearly answered. Existing premises suggest that, under different circumstances, either higher or lower IBPV could contribute to postoperative complications. Taking into account the heterogeneity and quality of the studies, the conclusions may not be definitive and further well-designed studies are needed to clarify this unclear relationship.
Supplementary Information The online version contains supplementary material available at https://doi.org/10.1007/s00540-022-03035-w.

Acknowledgements Registration and protocol: We did not register a protocol for this review.

Author contributions All the authors contributed to the study conception and design. Material preparation, data collection, and analysis were performed by ZP, MC, and $Ł J K$. The first draft of the manuscript was written by $\mathrm{ZP}$ and MC. All the authors commented on previous versions of the manuscript. All the authors read and approved the final manuscript.

Funding This research did not receive any specific grant from funding agencies in the public, commercial, or not-for-profit sectors.

\section{Declarations}

Conflict of interest The authors have no conflicts of interest.

Open Access This article is licensed under a Creative Commons Attribution 4.0 International License, which permits use, sharing, adaptation, distribution and reproduction in any medium or format, as long as you give appropriate credit to the original author(s) and the source, provide a link to the Creative Commons licence, and indicate if changes were made. The images or other third party material in this article are included in the article's Creative Commons licence, unless indicated otherwise in a credit line to the material. If material is not included in the article's Creative Commons licence and your intended use is not permitted by statutory regulation or exceeds the permitted use, you will need to obtain permission directly from the copyright holder. To view a copy of this licence, visit http://creativecommons.org/licenses/by/4.0/.

\section{References}

1. Sessler DI, Bloomstone JA, Aronson S, Berry C, Gan TJ, Kellum JA, Plumb J, Mythen MG, Grocott MPW, Edwards MR, Miller TE, Perioperative Quality Initiative-3 workgroup, POQI chairs, Miller TE, Mythen MG, Grocott MP, Edwards MR, Physiology group, Preoperative blood pressure group, Intraoperative blood pressure group, Postoperative blood pressure group. Perioperative quality initiative consensus statement on intraoperative blood pressure, risk and outcomes for elective surgery. Br J Anaesth. 2019;122(5):563-74. https://doi.org/10.1016/j.bja.2019.01.013.

2. Kristensen SD, Knuuti J, Saraste A, Anker S, Bøtker HE, Hert SD, Ford I, Gonzalez-Juanatey JR, Gorenek B, Heyndrickx GR, Hoeft A, Huber K, Iung B, Kjeldsen KP, Longrois D, Lüscher TF, Pierard L, Pocock S, Price S, Roffi M, Sirnes PA, Sousa-Uva M, Voudris V, Funck-Brentano C. Authors/Task Force Members 2014 ESC/ESA guidelines on non-cardiac surgery: cardiovascular assessment and management: the Joint Task Force on non-cardiac surgery: cardiovascular assessment and management of the European Society of Cardiology (ESC) and the European Society of Anaesthesiology (ESA). Eur Heart J. 2014;35(35):2383-431. https://doi.org/10.1093/eurheartj/ehu282.

3. Wesselink EM, Kappen TH, Torn HM, Slooter AJC, van Klei WA. Intraoperative hypotension and the risk of postoperative adverse outcomes: a systematic review. Br J Anaesth. 2018;121(4):70621. https://doi.org/10.1016/j.bja.2018.04.036.

4. Page MJ, McKenzie JE, Bossuyt PM, Boutron I, Hoffmann TC, Mulrow CD, Shamseer L, Tetzlaff JM, Akl EA, Brennan SE, Chou 
R, Glanville J, Grimshaw JM, Hróbjartsson A, Lalu MM, Li T, Loder EW, Mayo-Wilson E, McDonald S, McGuinness LA, Stewart LA, Thomas J, Tricco AC, Welch VA, Whiting P, Moher D. The PRISMA 2020 statement: an updated guideline for reporting systematic reviews. BMJ. 2021;29(372): n71. https://doi.org/10. 1136/bmj.n71.

5. Wells GA, Shea B, O'Connell D, Peterson J, Welch V, Losos M, Tugwell P. The Newcastle-Ottawa Scale (NOS) for assessing the quality of non-randomized studies in meta-analysis. 2000. http://www.ohri.ca/programs/clinical_epidemiology/oxford.asp. Accessed 30 Apr 2021.

6. Viswanathan M, Ansari MT, Berkman ND, Chang S, Hartling L, McPheeters M, Santaguida PL, Shamliyan T, Singh K, Tsertsvadze A, Treadwell JR. Assessing the risk of bias of individual studies in systematic reviews of health care interventions. 2012 Mar 8. In: methods guide for effectiveness and comparative effectiveness reviews. Rockville (MD): Agency for Healthcare Research and Quality (US); 2008. https://www.ncbi.nlm.nih.gov/ books/NBK91433/. Accessed 30 Apr 2021.

7. Neuner B, Weiss B, Wulfekammer T, Radtke F, Franck M, Spies C. Absolute values of blood pressure fluctuation as an independent risk factor for postoperative delirium: secondary, exploratory analysis of the randomized controlled "surgery depth of anaesthesia and cognitive outcome"-(SuDoCO) trial. J Am Coll Cardiol. 2016;68(16):C185.

8. James LA, Levin MA, Lin HM, Deiner SG. Association of preoperative frailty with intraoperative hemodynamic instability and postoperative mortality. Anesth Analg. 2019;128(6):1279-85. https://doi.org/10.1213/ANE.0000000000004085.

9. Zevallos CB, Dai B, Dandapat S, Quispe-Orozco D, Holcombe A, Ansari S, Farooqui M, Derdeyn CP, Samaniego EA, OrtegaGutierrez S. Greater intraprocedural systolic blood pressure and blood pressure variability are associated with contrast-induced neurotoxicity after neurointerventional procedures. J Neurol Sci. 2021;420: 117209. https://doi.org/10.1016/j.jns.2020.117209.

10. Li J, Zhao Y, Zhao M, Cao P, Liu X, Ren H, Zhang D, Zhang Y, Wang R, Zhao J. High variance of intraoperative blood pressure predicts early cerebral infarction after revascularization surgery in patients with Moyamoya disease. Neurosurg Rev. 2020;43(2):759-69. https://doi.org/10.1007/s10143-019-01118-z.

11. Radinovic K, Markovic Denic L, Milan Z, Cirkovic A, Baralic M, Bumbasirevic V. Impact of intraoperative blood pressure, blood pressure fluctuation, and pulse pressure on postoperative delirium in elderly patients with hip fracture: a prospective cohort study. Injury. 2019;50(9):1558-64.

12. Park S, Lee HC, Jung CW, Choi Y, Yoon HJ, Kim S, Chin HJ, Kim M, Kim YC, Kim DK, Joo KW, Kim YS, Lee H. Intraoperative arterial pressure variability and postoperative acute kidney injury. Clin J Am Soc Nephrol. 2020;15(1):35-46. https://doi.org/ 10.2215/CJN.06620619.

13. Wiórek A, Krzych ŁJ. Intraoperative blood pressure variability predicts postoperative mortality in non-cardiac surgery-a prospective observational cohort study. Int J Environ Res Public Health. 2019;16(22):4380. https://doi.org/10.3390/ijerph1622 4380.

14. Prasad V, Toschi N, Canichella A, Marcellucci M, Coniglione F, Dauri M, Guerrisi M, Heldt T. Intraoperative hemodynamics predict postoperative mortality in orthotopic liver transplantation. Annu Int Conf IEEE Eng Med Biol Soc. 2015;2015:989-92. https://doi.org/10.1109/EMBC.2015.7318530.

15. Mascha EJ, Yang D, Weiss S, Sessler DI. Intraoperative mean arterial pressure variability and 30-day mortality in patients having noncardiac surgery. Anesthesiology. 2015;123(1):79-91. https://doi.org/10.1097/ALN.0000000000000686.

16. Cai YH, Wang HT, Zhou JX. Perioperative predictors of extubation failure and the effect on clinical outcome after infratentorial craniotomy. Med Sci Monit. 2016;22:2431-8. https://doi.org/10. 12659/msm.899780.

17. Levin MA, Fischer GW, Lin HM, McCormick PJ, Krol M, Reich DL. Intraoperative arterial blood pressure lability is associated with improved 30 day survival. Br J Anaesth. 2015;115(5):71626. https://doi.org/10.1093/bja/aev293.

18. Hansen TW, Thijs L, Li Y, Boggia J, Kikuya M, Björklund-Bodegård K, Richart T, Ohkubo T, Jeppesen J, Torp-Pedersen C, Dolan E, Kuznetsova T, Stolarz-Skrzypek K, Tikhonoff V, Malyutina S, Casiglia E, Nikitin Y, Lind L, Sandoya E, Kawecka-Jaszcz K, Imai Y, Wang J, Ibsen H, O'Brien E, Staessen JA, International Database on Ambulatory Blood Pressure in Relation to Cardiovascular Outcomes Investigators. Prognostic value of reading-to-reading blood pressure variability over 24 hours in 8938 subjects from 11 populations [published correction appears in Hypertension. 2010 Jun;55(6):e27]. Hypertension. 2010;55(4):1049-57. https://doi. org/10.1161/HYPERTENSIONAHA.109.140798.

19. Collange O, Jazaerli L, Lejay A, Biermann C, Caillard S, Moulin B, Chakfe N, Severac F, Schaeffer M, Mertes PM, Steib A. Intraoperative pleth variability index is linked to delayed graft function after kidney transplantation. Transplant Proc. 2016;48(8):261521. https://doi.org/10.1016/j.transproceed.2016.06.04.

20. Nolan J, Batin PD, Andrews R, Lindsay SJ, Brooksby P, Mullen M, Baig W, Flapan AD, Cowley A, Prescott RJ, Neilson JM, Fox KA. Prospective study of heart rate variability and mortality in chronic heart failure: results of the United Kingdom heart failure evaluation and assessment of risk trial (UK-heart). Circulation. 1998;98(15):1510-6. https://doi.org/10.1161/01.cir.98.15.1510.

21. Neukirchen M, Kienbaum P. Sympathetic nervous system: evaluation and importance for clinical general anesthesia. Anesthesiology. 2008;109(6):1113-31. https://doi.org/10.1097/ALN.0b013 e31818e435c.

Publisher's Note Springer Nature remains neutral with regard to jurisdictional claims in published maps and institutional affiliations. 